\title{
Topography of genetic loci in tissue samples: towards new diagnostic tool using interphase FISH and high-resolution image analysis techniques
}

\author{
I. Koutná ${ }^{\mathrm{a}}$, S. Kozubek ${ }^{\mathrm{b}, *}$, J. Žaloudík $^{\mathrm{c}}$, \\ M. Kozubek ${ }^{a}$, E. Lukášová ${ }^{b}$, Pa. Matula ${ }^{a}$, \\ E. Bártová ${ }^{\mathrm{b}}$, M. Skalníkováa ${ }^{\mathrm{a}}$, A. Cafourková ${ }^{\mathrm{b}}$ \\ and P. Jirsová ${ }^{\mathrm{b}}$ \\ ${ }^{\text {a }}$ Faculty of Informatics, Masaryk University, \\ Botanická 68a, 60200 Brno, Czech Republic \\ ${ }^{\mathrm{b}}$ Institute of Biophysics, Academy of Sciences, \\ Královopolská 135, 61265 Brno, Czech Republic \\ ${ }^{\mathrm{c}}$ Faculty Hospital of Masaryk University, Bohunice, \\ 63500 Brno, Czech Republic
}

Received April 2000

Accepted 26 June 2000

Using single and dual colour fluorescence in situ hybridisation (FISH) combined with image analysis techniques the topographic characteristics of genes and centromeres in nuclei of human colon tissue cells were investigated. The distributions of distances from the centre-of-nucleus to genes (centromeres) and from genes to genes (centromeres to centromeres) were studied in normal colon tissue cells found in the neighbourhood of tumour samples, in tumour cell line HT-29 and in promyelocytic HL-60 cell line for comparison. Our results show that the topography of genetic loci determined in 3D-fixed cell tissue corresponds to that obtained for 2D-fixed cells separated from the tissue. The distributions of the centre-of-nucleus to gene (centromere) distances and gene to gene (centromere to centromere) distances and their average values are different for various genetic loci but similar for normal colon tissue cells, HT-29 colon tumour cell line and HL-60 promyelocytic cell line. It suggests that the arrangement of genetic loci in cell nucleus is conserved in different types of human cells. The investigations of trisomic

\footnotetext{
${ }^{*}$ Corresponding author: Stanislav Kozubek, Institute of Biophysics, Academy of Sciences, Královopolská 135, 61265 Brno, Czech Republic. Tel.: +420 54151 7139; Fax: +420 54124 0498; E-mail: kozubek@ibp.cz.
}

loci in HT-29 cells revealed that the location of the third genetic element is not different from the location of two homologues in diploid cells. We have shown that the topographic parameters used in our experiments for different genetic elements are not tissue or tumour specific. In order to validate high-resolution cytometry for oncology, further investigations should include more precise parameters reflecting the state of chromatin in the neighbourhood of critical oncogenes or tumour suppresser genes.

\section{Introduction}

Interphase chromatin is a reliable morphological indicator of the physiological cell condition: processes such as proliferation, differentiation, transformation, apoptosis, etc. are usually correlated with specific chromatin changes [3,4,31]. A quantitative evaluation of the chromatin pattern is based either on the separate analysis of each single structural attribute (size, shape, density, arrangement and distribution of chromatin grains) or on a global evaluation. The global evaluation of the chromatin patterns may be obtained by considering the chromatin structure as a coherent texture (heterogeneity, granularity, condensation, radial distribution) [9]. Measurements of the chromatin patterns by microscopy are based on image processing. DNA image analysis is frequently performed in clinical practise as a prognostic tool and for the improvement of diagnostics in some cancers cases [27]. Chromatin texture is determined by the global topographic arrangement of cell nuclei and represent an elementary histological characteristic of tumour tissue. For this reason chromatin texture plays also a central role in diagnosis and grading of malignancy by the pathologist, and an understanding of its principles is of utmost importance for tumour biology [24]. The analy- 
sis of the nuclear chromatin texture opens up a new field for associating morphology with functional properties [33].

FISH methods are frequently used in diagnostics of some cancers, in most cases in the diagnostics of heamoblastoses. For example, the $\mathrm{t}(9 ; 22)(\mathrm{q} 34 ; 11)$ translocation leads to the formation of chimeric ABLBCR gene, which plays a crucial role in the pathogenesis of chronic myeloid leukaemia (CML) [15]. FISH has been also successfully used in diagnostics of solid tissue tumours. Aberrations of chromosome 1 detected in breast cancer using dual colour FISH correlated with protein activity of c-erbB-2 and bcl-2 [10]. The combination of FISH and image analysis provides a new tool for the measurement of topographic parameters [16]. The recent studies document a structured arrangement of chromosomal domains in interphase nuclei. During the interphase, each chromosome occupies a distinct domain within the nucleus [5,23]. Chromosomes or genes are located in a restricted volume of the nucleus. The proximity of chromosome territories can influence the frequency of their specific structural rearrangements under certain circumstances [18]. It was shown that exchange aberrations are very frequent between the specific pairs of chromosomes in lymphocytes irradiated with fast neutrons [21]. These results indicate that chromosomes located well apart will interact with less probability.

The topographic arrangement of cell nuclei arises as a result of positioning of individual chromosomes and genes. The topographic parameters of specific genes or chromosomes represent obviously more elementary morphological indicator in comparison to the chromatin texture. The location of chromosomes, genes and other specific DNA sequences can be visualised using FISH in both single cells and tissue sections. Topographic parameters are important not only in relation to the induction of translocation. Experiments with Drosophila and yeast show, that also gene expression might be influenced by its position [7,26]. Epigenetic regulation of gene expression does not probably play an important role within the cell cycle but it could be very important in differentiation, apoptosis and also in malignant transformation. For example, in differentiated HL-60 cells, c-myc is relocated closer to the nuclear periphery and its expression is downregulated. The fact that both processes have common kinetics during granulocytic differentiation support the idea that the relocation of the c-myc gene to the nuclear periphery and the condensation of the chromosome 8 domains might be associated with the c-myc gene silencing [2].
Positions of chromosomes, centromeres or individual genetic loci reveal non-random distribution of these genetic elements in the cell nucleus. Several studies show that the centromeres $[8,13,14]$ or oncogenes $[21$, 22] are distributed non-randomly in the nuclear volume, at least in some stages of the cell cycle. Distinctly non-random chromosome organisation was found in $\mathrm{G}_{1}$ human lymphocytes with several centromeric regions localised on the nuclear periphery and telomeric regions in the interior of the cell nucleus [11]. The higher order nuclear structure have been investigated using both human blood cells (lymphocytes, bone marrow) and cell cultures (HL-60, U-937, ML-1, K-562) $[1,19,20]$. To our best knowledge, the topographic parameters in solid tissue have not been studied yet. The main reason is the relative simplicity of the methods of separating, fixing and, what is very important, evaluating the results in individual cells in comparison with tissues. However, about $80 \%$ from all malignancies are solid tumours and, therefore, the detailed studies of the topographic parameters of cell nuclei separated directly from the human donor tissues are needed. Differences in the topography of genetic elements between normal and malignant tissue can be associated with malignant transformation, which can be of great value for diagnostics. Visualisation of specific oncogenes, tumour suppressor genes, their neighbourhood and the determination of appropriate parameters might have diagnostic importance and eventually could help in therapy.

In this study, the topographic parameters of specific genetic loci (genes and centromeres) were investigated in normal colon tissue separated from a colorectal carcinoma after the biopsy. These parameters were compared with those on the HT-29 human colon cell line and the HL-60 human promyelocytic cell line cultured in laboratory conditions. We show that the spatial distributions of genes and centromeres are very similar in nuclear volume of all cell types. The results indicate the existence of general principles of the nuclear organisation for cells growing in the cell suspension and cells forming a part of solid tissue. This work is a first step towards studying and formulating principles of chromatin organisation in solid tissue and the use of these results in diagnostics and therapy.

\section{Materials and methods}

\subsection{Tissue samples}

Fresh colon tissue was taken from five patients who underwent the surgery of colorectal carcinoma at Fac- 
ulty hospital Bohunice of Masaryk University. Immediately after resection material was immersed into the culture flask with $10 \mathrm{ml}$ of RPMI-medium containing $15 \%$ of FCS (PAN, Germany) and $15 \mathrm{U} / \mathrm{ml}$ of heparin. After the histopathological examination, parts of tissue without neoplastic lesion were selected for studying gene location.

\subsection{Cell preparation and fixation}

Tissue samples was rinsed with phosphate buffer saline (PBS) in 3 exchanges an digested in freshly prepared $0.5 \%$ pepsin (Sigma) in $0.9 \% \mathrm{NaCl}$ at $37^{\circ} \mathrm{C}$ for $15 \mathrm{~min}$. This was followed by desintegration of tissue and filtration of free cells through a $25 \mu \mathrm{m}$ pore size nylon fibre Sefar-Nitex (Sefar, Switzerland). Free cells were pelleted down by centrifugation and washed in PBS at $4^{\circ} \mathrm{C}$. For 2D fixation of nuclei we used the routine procedure including fixation in $(1: 3)$ acetic acid/methanol solutions. 3D fixation was done with $4 \%$ paraformaldehyde as described earlier [17, 25].

\subsection{Preparation of tissue sections for FISH}

A piece of tissue was immersed in $10 \%$ paraformaldehyde for $20 \mathrm{~min}$, washed in PBS $(3 \times 5 \mathrm{~min})$ and embedded in medium for frozen tissue (Histolab). Frozen tissue was cut to section of $4 \mu \mathrm{m}$ at $-30^{\circ} \mathrm{C}$ and mounted on poly-L-lysine-coated slides. Before FISH analysis slides were covered with $0.2 \%$ pepsin solution, $\mathrm{pH} 1.5$, incubated $20 \mathrm{~min}$ at $37^{\circ} \mathrm{C}$ and washed in PBS containing $0.1 \%$ Triton-X-100 $(3 \times 5 \mathrm{~min})$ and PBS $(1 \times 5 \mathrm{~min})$. Slides were then immersed in $0.1 \%$ paraformaldehyde for $10 \mathrm{~min}$, washed and left dry on the air.

\subsection{Cell culture}

The human promyelocyte HL-60 cell line and human adenocarcinoma HT-29 colon tissue cell line grew up 25 passage in RPMI-medium supplemented with $10 \%$ foetal calf serum (PAN, Germany). The cells were cultured at $37^{\circ} \mathrm{C}$ in humidified atmosphere containing $5 \% \mathrm{CO}_{2}$. Cytological preparations were made by the routine procedure including cell harvesting by centrifugation, hypotonic shock in $0.075 \mathrm{~mol} / \mathrm{K} \mathrm{KCl}$ and fixation in $(1: 3)$ acetic acid/methanol solutions or by paraformaldehyde (4\%).

\subsection{Fluorescence in situ hybridisation (FISH)}

FISH was performed with (a) unique sequences of ABL, BCR (dual colour probe), c-MYC (Oncor, Appligene), (b) alpha-satellite DNA probes of centromeres modified by digoxigenin (Oncor, Appligene). The procedure of hybridisation with these two different types of probes and post-hybridisation wash was performed according to the instructions of the manufacturers. Counterstaining was performed with DAPI $(0.02 \mu \mathrm{g} / \mathrm{ml})$ in vectashield (Vector).

\subsection{Image acquisition and analysis}

A high-resolution cytometer based on an automated inverted ZEISS Axiovert (Germany) microscope, a MicroMax (Princeton Instruments, USA), CCD camera and the confocal unit CARV (Atto corp., USA) were used. The cytometer acquires images from microscopic slides automatically. For 2D fixed nuclei, several slices (3-6) with an axial step of about $0.5-1 \mu \mathrm{m}$ were acquired for each field of view and maximum image was calculated in order to increase the depth of focus. Usually $>500$ images were stored overnight into the computer memory and subsequently analysed. The cytometer acquires images from microscopic slides automatically. For 3D fixed nuclei, several slices (10-21) with an axial step of about $0.3-0.5 \mu \mathrm{m}$ were acquired for each field of view and the maximum image was calculated in order to increase the depth of focus. The analysis was performed using FISH 2.0 software [16], which enables the detection of signals inside nuclei. The positions of centromeres and genes were calculated relative to the centre of gravity coordinates of the nucleus. The algorithms used for finding nuclei and signals were described earlier [16]. Briefly, the nuclei are found using simple thresholding where the threshold is determined by the analysis of the histogram of the counterstain image. The genes or centromeres are detected inside nuclei using gradual thresholding [16]. The signals are evaluated according to their intensity and size. The information about the signals is written into text files and analysed further in Sigma Plot (Jandel Scientific, CA). The centre-of-nucleus to signal distances are normalised to the local radius (radius in the direction of the signal). A number of user-defined options can be set for both acquisition and for analysis. For example, the reallocation and automated reacquisition are possible after repeated hybridisation for a set of images. 


\subsection{Manual segmentation of cell nuclei}

In the case of 3D fixed nuclei of tissue section we used manual segmentation for the determination of borders of nuclei. An automatic or semi-automatic methods were not used because automatic segmentation of cell nuclei in tissues is a hard problem and has not been satisfactory solved yet. Border of nucleus was created by human by entering several (typically 6-8) points of desired nucleus outline by clicking on mouse. When the last point was entered computer interpolated given points with a closed smooth curve. Piecewise cubic Bézier spline was used as interpolation curve. Construction of our interpolation curve is explained in the following paragraph.

Let $P_{1}, P_{2}, \ldots, P_{n}$ be $n$ entered points. Between two successive points $P_{i}$ and $P_{i+1}$ cubic Bézier curve with control points $P_{i}, R_{i}, L_{i+1}, P_{i+1}$ was created, where new control points $R_{i}, L_{i}$ were given by terms

$$
\begin{aligned}
& R_{i}=P_{i}+\frac{\left\|P_{i} P_{i+1}\right\|}{3\left\|P_{i-1} P_{i+1}\right\|} P_{i-1} P_{i+1}, \\
& L_{i}=P_{i}-\frac{\left\|P_{i} P_{i-1}\right\|}{3\left\|P_{i-1} P_{i+1}\right\|} P_{i-1} P_{i+1} .
\end{aligned}
$$

The $P_{i} P_{i+1}$ notation states for vector from point $P_{i}$ to point $P_{i+1}$ and $\left\|P_{i} P_{i+1}\right\|$ is Euclidean length of vector $P_{i} P_{i+1}$. If this curve didn't fit the nucleus border well it could be modified after interpolation by moving the control points. Bézier curves are often used in many graphics systems, because they are easy to edit and their rendering is quick.

\subsection{Statistical evaluation of the results}

The number of nuclei analysed in one topological analysis ranged between 200-1000. Experiments were repeated at least 2 times for each gene and each cell type. A high number of nuclei analysed in $2 \mathrm{D}$ provide information about 3D structure. The recalculation requires, however, random positioning of cell nuclei in preparations and spherical shape of the nuclei. These conditions are well fulfilled for cells fixed from cell suspension (HL-60 and HT-29). We used also parts of colon tissue without neoplastic lesion. Fixed nuclei of these samples preserve mostly relatively spherical shape.

The distributions of 2D projections $(P)$ of centreof-nucleus to signal fractional distances $(r)$ were transformed into 3D distributions assuming random orien- tation of cell nuclei (this condition is evidently fulfilled for cells growing in suspension). The procedure was described earlier $[2,18,20,22,29]$. Briefly, we take, for example, 10 layers (shells) in a sphere at 0-10\%, 10$20 \%, \ldots, 90-100 \%$ of radius. For each layer, the distribution of $2 \mathrm{D}$ projections of centre of the sphere to point fractional distances, $B(r)$, is calculated by Monte Carlo simulation (randomly generating points within the given layer). In such a way we obtain 10 distributions $B_{1}, B_{2}, \ldots, B_{10}$ corresponding to the layers 0 $10 \%, 10-20 \%, \ldots, 90-100 \%$ of radius. These distributions form approximate triangles restricted to the regions between zero and the radius of the layer (parallel projections cannot be longer than the original distance; they can be shorter). The $2 \mathrm{D}$ distribution $P(r)$ is written as a superposition of theoretical 2D distributions of the 10 layers:

$$
P(r)=\sum_{i=1}^{10} A_{i} B_{i}(r),
$$

where $A_{i}$ are coefficients obtained by fitting. The coefficients $A_{i}$ give the weights with which the individual layers are involved in $P(r)$ distribution; therefore, $A_{i}$ represent the $3 \mathrm{D}$ distribution of centre-ofnucleus to signal distances. For 10 layers $0-10 \%, 10-$ $20 \%, \ldots, 90-100 \%$ we have fractional distances $r=$ $0.05,0.15, \ldots, 0.95$ with coefficients $A_{1}, A_{2}, \ldots, A_{10}$.

Theoretical distributions of gene to gene and centromere to centromere distances were calculated using Monte-Carlo simulation according to the RS model [2, $18,20,22,29]$. The RS model is based on experimental finding that the radial centre to gene distributions are nonrandom (genes are located in certain spherical layers) and the basic assumption of this model is the random angular distribution of genes inside spherical layers. Therefore, the RS model represents a tool for testing the randomness of gene to gene distributions inside spherical layers. The results of the calculations according to the RS model are frequently in good agreement with experimental results suggesting the validity of the model in many cases.

The simulated calculation for 2 pairs of genes is usually performed. It is based on the centre to gene distributions for these genes as an input for the modulated random number generator: the probabilities of gene appearance in different layers (coefficients $A_{i}$ ) are taken as weights of the random number generator. 4 points representing 2 gene pairs are placed inside a sphere using the generator and their 2D projections are computed. The experimental centre to gene distributions 
are automatically reproduced owing to the construction of the generator. The distribution of gene to gene distances is calculated for homologous as well as for heterologous genes from $\approx 10^{5}$ calculations.

The results of our calculations might be affected by irregular shape of cell nuclei. However, owing to the fact that the distances are normalised to the local radius, the deviations are minimised. In the case of 2D fixation the calculations remain valid if the $2 \mathrm{D}$ projections normalised to the local radius correspond to the projections obtained in 3D fixed samples. We have found earlier [16] that experimental distributions obtained from 2D and 3D fixed samples are quite similar with deviations of the average values less than 10$15 \%$. The agreement between experimental and theoretical distributions in the case of $2 \mathrm{D}$ fixed samples is similar as compared to 3D fixed ones.

\section{Results}

The distributions of the centre-of-nucleus to signal and signal to signal 2D distances for the ABL, BCR, and c-MYC genes and chromosome 1, 8, and 9 centromeres were measured in human cells of colon tissue, in the HT-29 human colon cell line and, for comparison, in the HL-60 human promyelocytic cell line.

\subsection{Topography of cell nuclei in $3 D$ fixed tissue sections do not differ from $2 D$ fixed cells}

The topographic parameters were investigated either in $4 \mu \mathrm{m}$ thick tissue sections (Fig. 1) or in individual cells separated from a colorectal tissue. The comparison of the topographic parameters of the chromosome 7 centromere $\left(\mathrm{CC}_{7}\right.$ and $\left.\mathrm{C}_{7} \mathrm{C}_{7}\right)$ determined for $3 \mathrm{D}$ fixed colon tissue sections and 2D fixed nuclei of individual cells separated from the same colon tissue is shown in Fig. 2. The average values of the $C_{7}$ and $C_{7} C_{7}$ parameters obtained for $2 \mathrm{D}$ and $3 \mathrm{D}$ fixed HL-60 cells are compared in Table 1. As can be seen, the distributions of centre-of-nucleus to signal and signal to signal distances for 2D fixation are very similar to those obtained for 3D fixation in both cells grown in suspension and cells fixed in tissue sections. Similar results were obtained also for other genetic loci, however, the number of cell nuclei analysed was much larger for cells separated from the tissue owing to the fact that automated analysis was used in this case. Therefore, further results are presented for cells separated from the tissue and fixed by standard technique.

\subsection{Genes and centromeres are located in spherical layers at specific centre-of-nucleus to locus distance}

The distributions of the centre-of-nucleus to signal 2D distances for 3 genes and 3 centromeres were determined in 3 cell types (Fig. 3). The average values of these distributions are shown in Table 2. It was found that different genetic loci are positioned at the specific centre-of-nucleus to locus distances which are conserved in various cell types. The coding sequences of the ABL and BCR genes are located in the inner parts of the nuclear volume; the c-MYC gene is observed much closer to the nuclear membrane. Centromeric

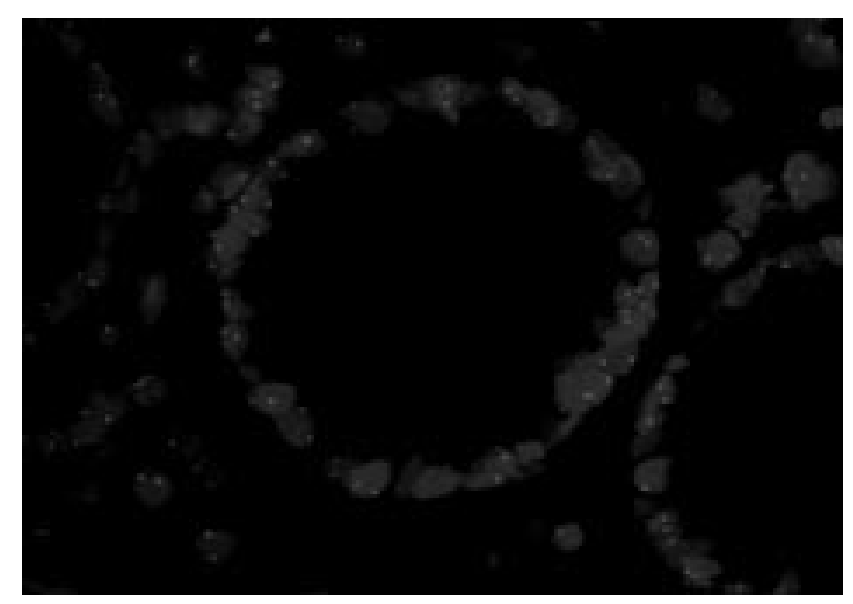

Fig. 1. Typical example of a two-dimensional image of 3D fixed, $4 \mu \mathrm{m}$ thick colon tissue section with FISH signals of chromosome 7 centromere. The nuclei were counterstained with DAPI. 


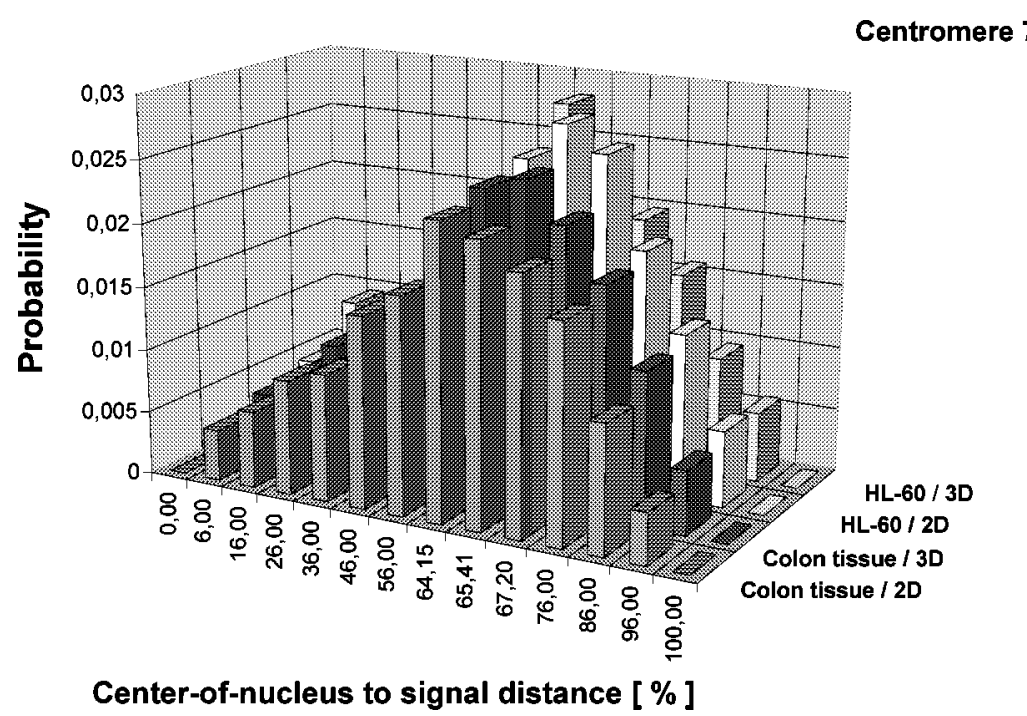

Fig. 2. Comparison of the distributions of the centre-of-nucleus to signal distances determined for the chromosome 7 centromere in 3D fixed, $4 \mu \mathrm{m}$ thick colon tissue sections with those in 2D fixed nuclei of individual cells separated from colon tissue and in HL-60 cells fixed in both 2D and 3D. The number of investigated cells was 200-300 for 3D analysis and 800-1000 for 2D analysis.

Table 1

Comparison of the average values and standard errors of centre-of-nucleus to signal and signal to signal distributions determined for chromosome 7 centromere in 2D fixed nuclei of individual cells separated from colon tissue and 3D fixed $4 \mu \mathrm{m}$ thick colon tissue sections

\begin{tabular}{|c|c|c|c|c|}
\hline \multirow[t]{3}{*}{$\overline{\text { Fixation }}$} & \multicolumn{4}{|c|}{ Cell type } \\
\hline & \multicolumn{2}{|c|}{ Colon tissue } & \multicolumn{2}{|c|}{ HL-60 } \\
\hline & $\overline{\overline{\mathrm{CN}_{7}}} \pm \mathrm{SE}[\%]$ & $\overline{\mathrm{N}_{7} \mathrm{~N}_{7}} \pm \mathrm{SE}[\%]$ & $\overline{\mathrm{CN}_{7}} \pm \mathrm{SE}[\%]$ & $\overline{\mathrm{N}_{7} \mathrm{~N}_{7}} \pm \mathrm{SE}[\%]$ \\
\hline $2 \mathrm{D}$ & $64.15 \pm 0.98$ & $88.80 \pm 2.38(87.39)^{*}$ & $65.15 \pm 0.94$ & $95.52 \pm 2.24(91.02)^{*}$ \\
\hline $3 \mathrm{D}$ & $66.44 \pm 2.02$ & $93.33 \pm 3.12(91.84)^{*}$ & $63.83 \pm 2.32$ & $91.17 \pm 2.26(89.96) *$ \\
\hline
\end{tabular}

The results obtained for nuclei of HL-60 cells fixed in both 2D and 3D are also shown for comparison. The number of investigated cells was 200-300 for 3D analysis and 800-1000 for 2D analysis.

$\overline{\mathrm{CN}_{7}}$ - average centre-of-nucleus to centromere 7 distance; $\overline{\mathrm{N}_{7} \mathrm{~N}_{7}}$ - average value of signal to signal distribution of homologous centromeres 7 (the distances are shown in \% of the nuclear radius, $R$ ); $\mathrm{SE}=\mathrm{SD} / \sqrt{n}$, where $n$ is the number of experiments (typical number is 3 ).

${ }^{*}$ Theoretical values calculated by Monte-Carlo simulations using experimentally determined centre to signal distributions (according to RS model).

heterochromatin is found near the nuclear membrane (centromeres 8 and 9), however, in some cases centromeres can be close to the centre-of-nucleus as some genes (e.g., centromere 1 and c-MYC). The sequence of the centre-of-nucleus to locus distances is as follows: $\mathrm{BCR} \approx \mathrm{ABL}<\mathrm{C} 1 \approx \mathrm{c}-\mathrm{MYC}<\mathrm{C} 8<\mathrm{C} 9$ for all cell types investigated.

\subsection{The distribution of genetic loci inside the spherical layers is random}

The signal to signal distributions are shown in Fig. 4 for 3 different loci (ABL, BCR, and c-MYC genes) and 3 cell types (HL-60, HT-29, and normal colon tissue cells). The experimental results are compared with the- oretical expectations based on the assumption of random positioning of genetic loci inside spherical layers (RS-model, see Materials and methods). Good correspondence between experimental points and theoretical curves suggests that the distribution of genes and centromeres inside the spherical layers is random (see also Table 3). The distributions obtained for HT-29 cells are narrower as compared to the other cell lines which reflects the fact that the cells are trisomic in all 3 genetic loci investigated.

\subsection{Positioning of the third genetic locus in trisomic cells}

We compared the nuclear localisation of the third genetic locus in cases of gene amplification (e.g., in 

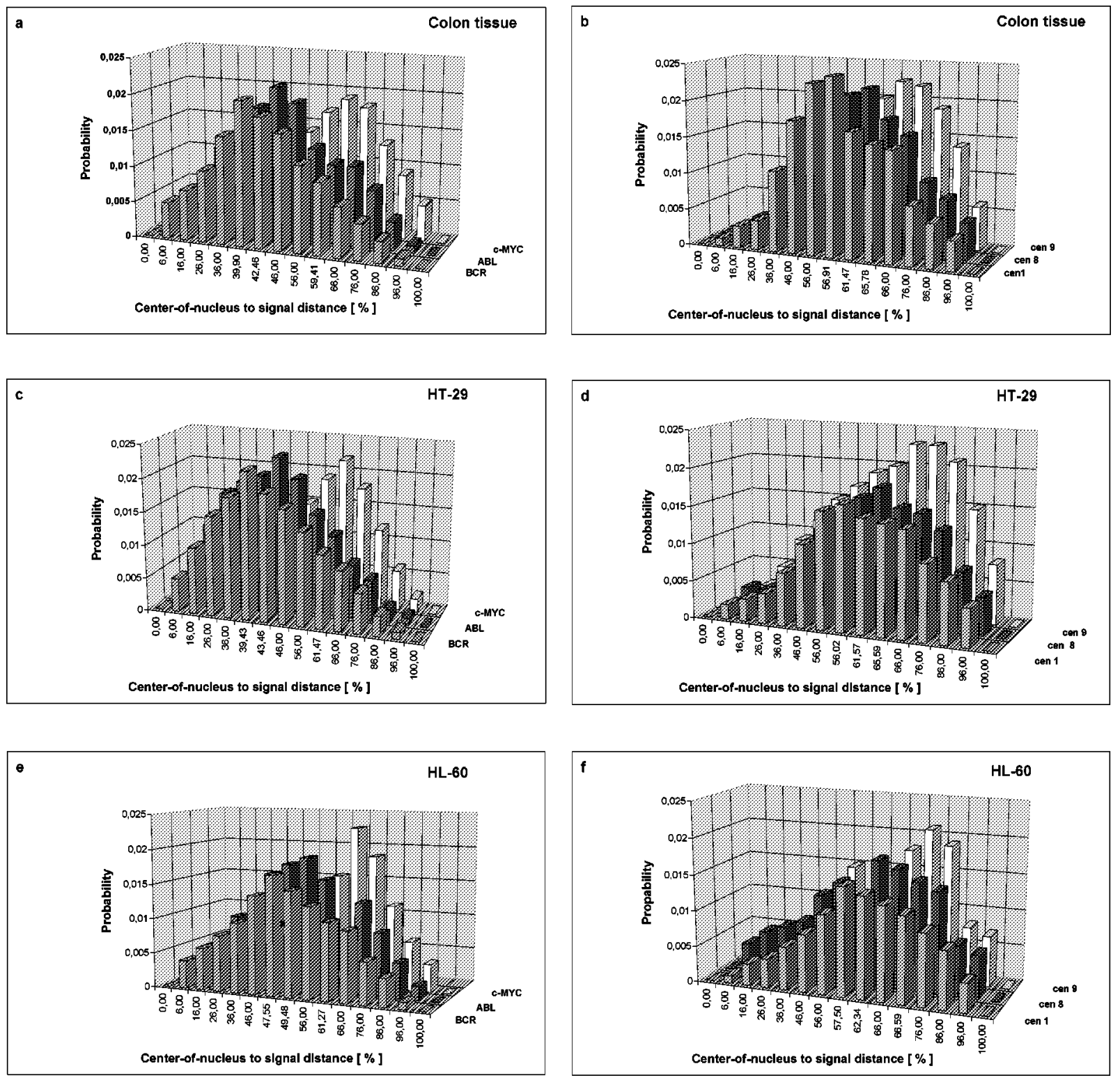

Fig. 3. Distributions of the centre-of-nucleus to signal distances determined for the ABL, BCR, and c-MYC genes (a, c, e) and the chromosome 1,8 , and 9 centromeres $(\mathrm{b}, \mathrm{d}, \mathrm{f})$ in nuclei of cells separated from colon tissue taken from surgery of colorectal carcinoma (a, b), HT-29 cells (c, d) and HL-60 cells $(e, f)$. The distributions are different for various genetic loci but similar for the cell lines investigated. The number of investigated cells was 800-1000 for each experiment.

HT-29 cells a number of loci are amplified). The average values of the centre-of-nucleus to locus distances for the ABL gene and chromosome 1 and 9 centromeres determined in HT-29 trisomic cells are compared with HL-60 cell line in Table 4. The positioning of all 3 genetic loci in HT-29 corresponds to those in HL-60 cells. In the case of the chromosome 1 centromere both 2 and 3 signals were observed in HT29 cell line. We analysed separately both populations of cells and compared the topographic distributions (Fig. 5). The centre-of-nucleus to centromere distributions are similar. The centromere to centromere distribution for HT-29 cells is narrower (mean values of the 3 signal to signal distances for each cell were evaluated), however, the average values of the distributions are similar for both 2 signal and 3 signal subpopulations. The theoretical lines obtained from the calculations based on the centre-of-nucleus to signal distri- 
Table 2

Average values and standard errors of centre-of-nucleus to signal 2D distributions for the ABL, BCR and c-MYC genes and chromosome centromeres 1,8 and 9

\begin{tabular}{|c|c|c|c|c|c|c|}
\hline \multirow[t]{2}{*}{ Cell type } & \multicolumn{6}{|c|}{ Genetic locus } \\
\hline & $\overline{\overline{\mathrm{CA}}} \pm \mathrm{SE}[\%]$ & $\overline{\mathrm{CB}} \pm \mathrm{SE}[\%]$ & $\overline{\mathrm{CM}} \pm \mathrm{SE}[\%]$ & $\overline{\mathrm{CN}_{1}} \pm \mathrm{SE}[\%]$ & $\overline{\mathrm{CN}_{8}} \pm \mathrm{SE}[\%]$ & $\overline{\mathrm{CN}_{9}} \pm \mathrm{SE}[\%]$ \\
\hline HL-60 & $49.49 \pm 0.32$ & $47.55 \pm 0.31$ & $61.27 \pm 0.58$ & $57.46 \pm 0.89$ & $62.34 \pm 0.89$ & $66.5 \pm 0.59$ \\
\hline HT-29 & $43.46 \pm 0.82$ & $39.42 \pm 0.79$ & $61.75 \pm 0.78$ & $56.05 \pm 1.40$ & $61.57 \pm 0.75$ & $65.59 \pm 1.31$ \\
\hline Colon tissue & $42.05 \pm 0.85$ & $39.90 \pm 0.86$ & $59.41 \pm 1.33$ & $56.91 \pm 1.33$ & $61.47 \pm 1.40$ & $65.78 \pm 0.96$ \\
\hline Mean values & $45.00 \pm 2.28$ & $42.29 \pm 2.63$ & $60.81 \pm 0.71$ & $56.80 \pm 0.41$ & $61.79 \pm 0.27$ & $65.99 \pm 0.31$ \\
\hline
\end{tabular}

The number of investigated cells was 800-1000 for each experiment. Average values and standard errors of 2-3 experiments were calculated.

$\overline{\mathrm{CA}}$ - average centre-of-nucleus to $\mathrm{ABL}$ distance; $\overline{\mathrm{CB}}$ - average centre-of-nucleus to BCR distance; $\overline{\mathrm{CM}}$ - average centre-of-nucleus to c-MYC distance; $\overline{\mathrm{CN}_{1}}$ - average centre-of-nucleus to centromere 1 distance; $\overline{\mathrm{CN}_{8}}$ - average centre-of-nucleus to centromere 8 distance; $\overline{\mathrm{CN}_{9}}-$ average centre-of-nucleus to centromere 9 distance (the distances are shown in $\%$ of the nuclear radius, $R$ ); $\mathrm{SE}=\mathrm{SD} / \sqrt{n}$, where $n$ is the number of experiments (typical number is 3 ).
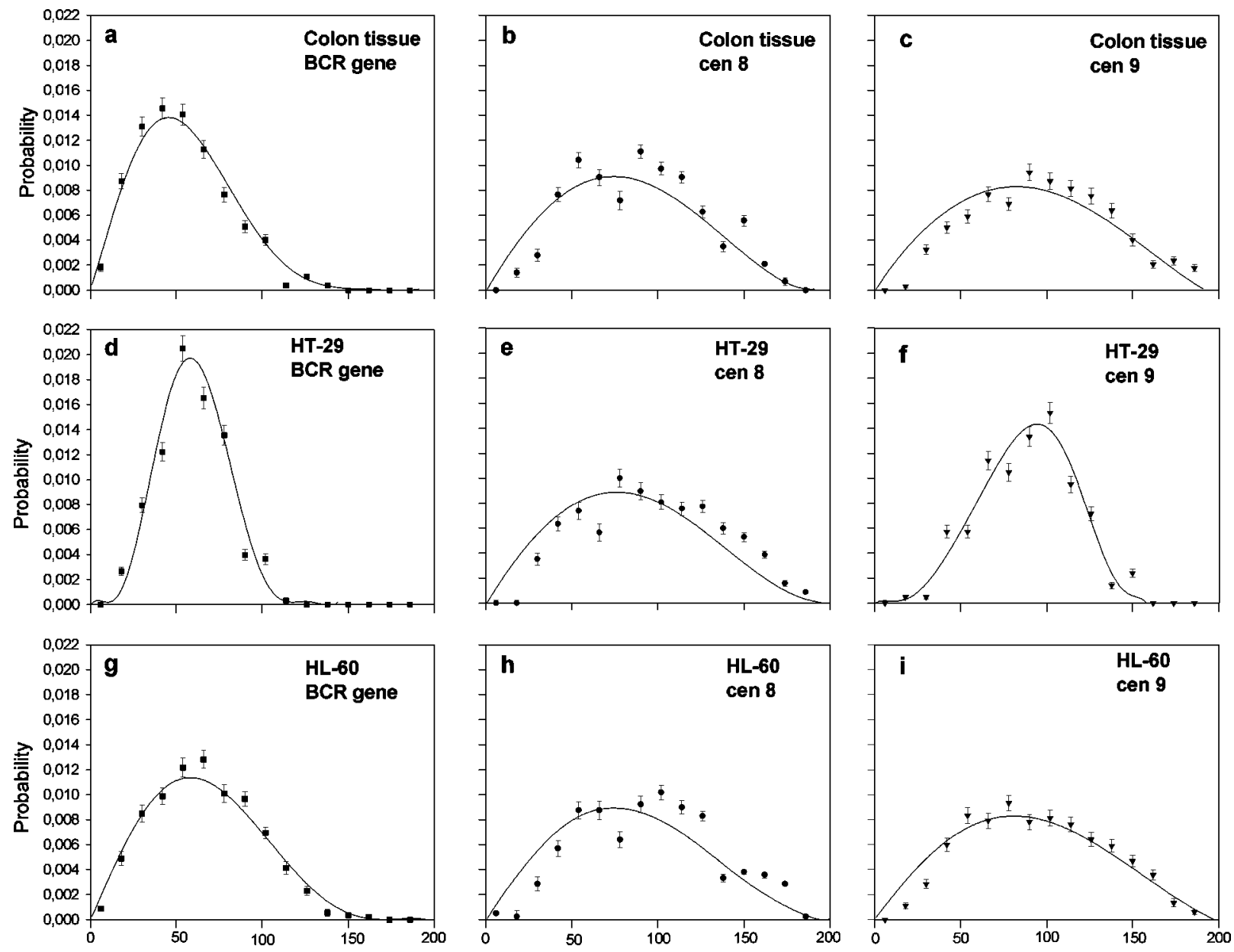

Signal to signal distance $[\%]$

Signal to signal distance [\%]

Signal to signal distance [\%]

Fig. 4. Distributions of the signal to signal distances determined for the homologous BCR genes (a, d, g), chromosome 8 (b, e, h) and 9 (c, f, i) centromeres in nuclei of cells separated from colon tissue of human donor (a, b, c), HT-29 (d, e, f) and HL-60 (g, h, i) cells. Experimental results are compared with theoretical functions (full curves) obtained by Monte-Carlo simulations (see Materials and methods). The number of investigated cells was $800-1000$ for each experiment. 

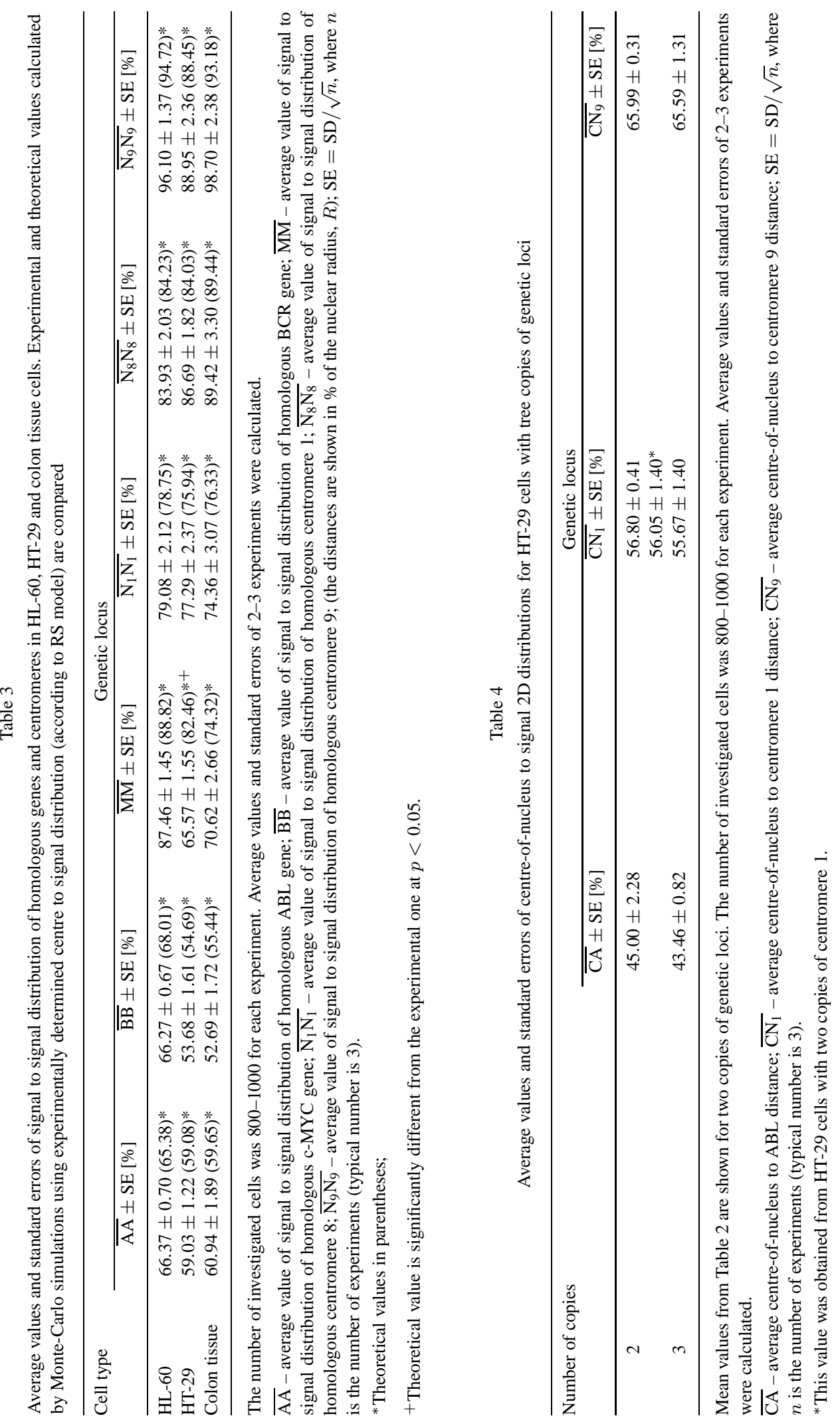

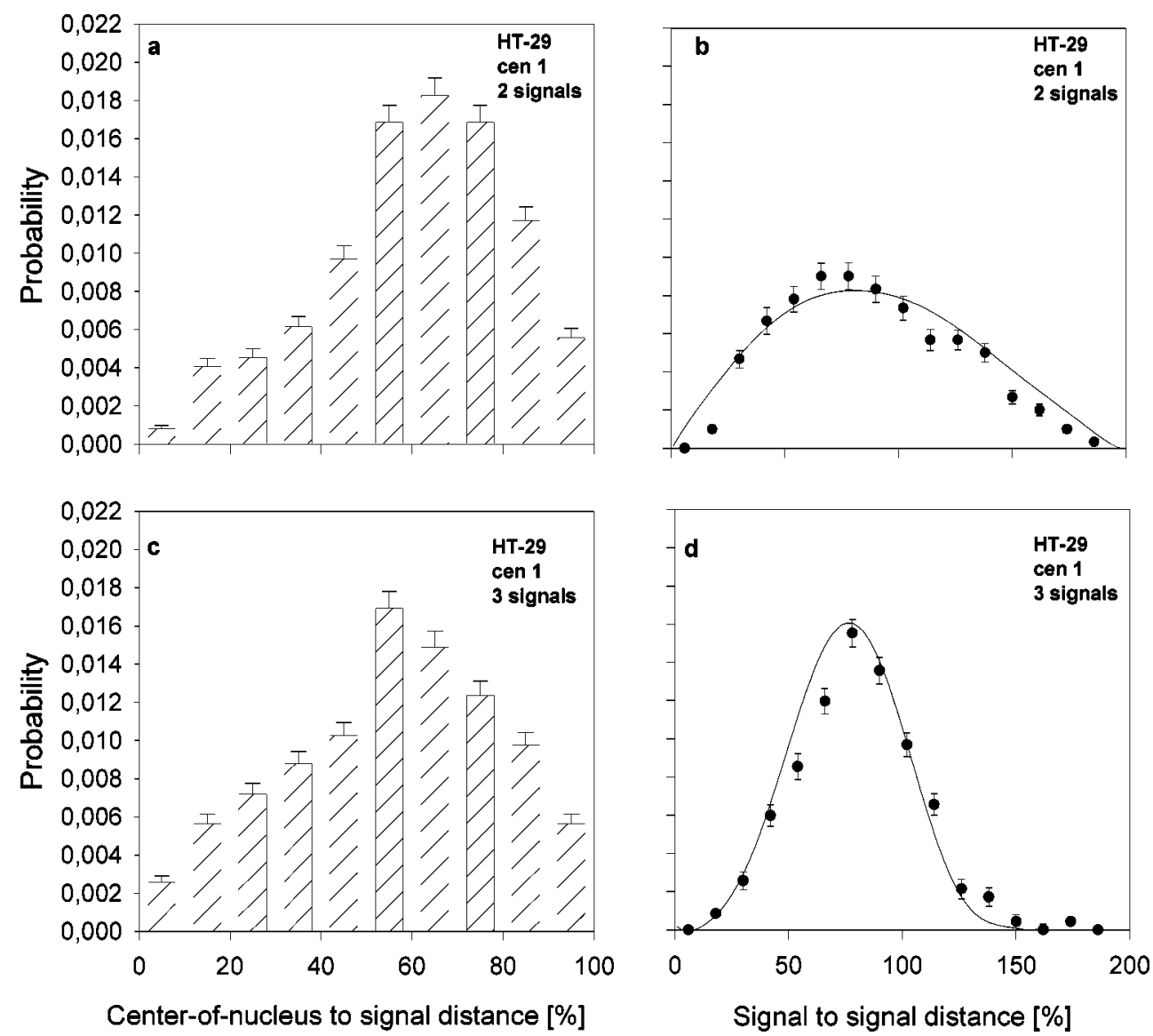

Fig. 5. Centre-of-nucleus to centromere 1 (a, c) and centromere to centromere (b, d) distributions determined in nuclei of HT-29 cells with two (a, b) or three (c, d) chromosomes 1. Experimental results are compared with theoretical functions (full curves) obtained by Monte-Carlo simulations (see Materials and methods). The number of investigated cells was 800-1000 for each experiment.

butions (see Materials and methods) correspond to the experimental points suggesting random positioning of the additional third centromere in the spherical layer specific for the given locus.

\section{Discussion}

Using FISH and image analysis techniques the nuclear topography of genes and centromeres for human colon cells in (1) tissue sections, (2) individual cells separated from the colon tissue and (3) in HT-29 tumour cell line isolated from the colon cancer was investigated. The results were compared to those obtained for HL-60 promyelocytic cell line. To our best knowledge, it is for the first time that the topography of the genetic material in tissue sections was investigated (Fig. 1) and it was shown that such kind of analysis is feasible. The images of the $3 \mathrm{D}$-fixed tissue samples were acquired in sections using confocal microscopy. Subsequently, the maximum image was calculated in order to increase the depth of focus, which resulted in bright and clear signals. Manual segmentation was used in order to separate cell nuclei found in close neighbourhood. 2D-analysis of 3D-fixed samples is frequently used in our experiments owing to much easier acquisition (a large number of maximum images can be automatically acquired overnight and stored to computer memory) and owing to the fact that large number of 2D-projections provide information on the real 3D-structure [16].

We have shown that the distributions of the centreof-nucleus to signal and signal to signal distances determined for cells in 3D-fixed tissue sections are very similar to those of 2D fixed cells separated from the tissue (Fig. 2 and Table 1). The image acquisition and analysis, particularly the image segmentation, are much easier for cells separated from the tissue and con- 
sequently much better statistics and more precise results can be achieved.

The positioning of genes and centromeres investigated in our experiments show that coding sequences of ABL and BCR genes are located mostly in the inner parts of the cell nucleus, whereas the positioning of the c-MYC gene is more peripheral. On the other hand, the heterochromatin of centromeres is located mostly near the nuclear membrane; in some cases (centromere 1) more central positioning was observed. The sequence of the genetic elements location from the centre to the membrane in cell nucleus $(\mathrm{ABL} \approx \mathrm{BCR}<\mathrm{Cen} 1<\mathrm{c}$ $\mathrm{MYC} \approx \mathrm{Cen} 8<\mathrm{Cen} 9)$ is conserved for all 3 cell types investigated (Fig. 3). These observations are in agreement with findings of Ferreira and Sadoni [12,28]. These authors distinguish euchromatic (transcriptionally active and early replicated) and heterochromatic (transcriptionally inactive and late replicated) higherorder compartments in the nucleus. Our results suggest that these compartments have some further internal structure. Various genetic loci are positioned inside spherical layers at some locus-specific centre-ofnucleus to locus distance.

On the basis of these and other our results [29] it can be concluded that euchromatic regions (coding sequences) are located mostly (but not exclusively) in the inner parts of the cell nucleus (ABL, BCR, IGH, TP53); heterochromatic regions are located mostly near the nuclear membrane (centromeres 8, 9, 18). This polar orientation of chromosome territories can be clearly seen in most chromosomes and most cell types investigated. There are chromosomes with and without pronounced polar orientation (chromosomes 9 and 8 , respectively) which is obviously not related to the molecular weights of chromosomes. For example, the differences between the positions of c-MYC and centromere 8 are not significant in most cell types. Such chromosomes will be called non-polar as opposite to the polar chromosomes (e.g., chromosome 9). The different degree of polar orientation of these 2 chromosomes with approximately the same molecular weight might be determined by the euchromatin content of these chromosomes.

Chromosome territories with high euchromatin content are located near the nuclear centre (e.g., chromosomes 9, 19) and chromosomes with low euchromatin content are located near the nuclear membrane [6,29]. It can be, therefore, expected that the positions of the coding sequences (genes) will reflect the positions of the chromosomes. Consequently, the composition of chromosomes (euchromatin to heterochromatin ratio) is responsible for chromosome polarity and its position in cell nucleus; the positions of individual genes might be subordinated to their neighbouring composition and to the composition of the whole chromosome.

As can be seen from Fig. 4, the distributions of signal to signal 2D distances clearly correspond to the random positioning of genetic loci investigated inside the spherical layers. These results were obtained using the model of rotational symmetry (RS-model). In some cases, however, 3D positions were determined and 3D distributions were calculated. 3D measurements confirmed the conclusions obtained from 2D.

The agreement between the theoretical and experimental gene to gene (centromere to centromere) distributions of distances seems to contradict with the spatial exclusiveness of chromosome territories. It could be expected that shorter distances between genes (centromeres) which are located in the inner parts of territories would be rare owing to nonzero territory dimensions. In fact, our measurements are not precise enough to determine very short distances for signals of the same colour (the first point in plots of Fig. 4 represents about $0.5 \mu \mathrm{m}$ ). However, in the case of 2 genes located on different chromosomes the resolution is much better and shorter distances can be measured. Experimental values are frequently higher as compared with theoretical predictions $[17,20]$, which suggests that chromosome territories do not prevent some genes to be located quite near to each other. It is in agreement with marked variability of chromosome territories and also with recent findings [31] suggesting that the territories are not homogenous structures (e.g., transcription sites are scattered throughout the territory).

The locus-specific centre-of-nucleus to locus distance remains conserved for various human cell types (Fig. 3) and also for the third copy of the locus (centromere 1 and 9) (Table 4, Fig. 5). The third copy is placed in the same layer as the first 2 loci and its positioning inside the layer is also random. Similar observations were made also for 4 centromeres of chromosome 9 in ML- 1 cells and for ABL/BCR loci in K562 cells where multiple copies of these genes were observed (own unpublished observation). This finding shows that the mechanism which localises genes and centromeres at some centre-of-nucleus to locus distance is independent of the number of loci. A straightforward explanation is that there are MARs/SARs sequences near each locus which address the position of the locus in cell nucleus [32]. Such mechanism would also ensure the same accessibility of the genetic locus to expression of additional loci. 


\section{Conclusions}

Topographic parameters of genes or chromosomes in cell nuclei of 3D-fixed tissue sections can be determined using recently developed FISH and image analysis techniques. The centre-of-nucleus to locus and locus to locus distances obtained from tissue sections of colon are similar to those obtained from cells separated from the tissue.

The positions of genes and centromeres in cells derived from colon tissue are locus-specific, however, they are conserved for all cell types investigated and similar to human blood cells. The ABL and BCR genes are located in the central parts of cell nucleus, c-MYC is located close to nuclear membrane. Centromeric heterochromatin is found mostly near the nuclear membrane. The position and orientation of the third chromosome in trisomic cells is similar to the position of these loci in diploid cells.

The topographic parameters used in our experiments (centre-of-nucleus to locus and locus to locus distances) are not tissue or tumour specific. In order to validate high-resolution cytometry for oncology, further investigations should include more precise parameters reflecting the state of chromatin in the neighbourhood of critical oncogenes or tumour suppressor genes.

\section{Acknowledgements}

This work was supported by the Grant Agency of the Ministry of Education of the Czech Republic (Grant No. VS-97031), Ministry of Health of the Czech Republic (Grant No. NM 15-3 and NM 5955) and by the Grant Agency of the Czech Republic (Grant No. 524/98/0190).

\section{References}

[1] E. Bártová, S. Kozubek, M. Kozubek et al., The influence of the cell cycle, differentiation and irradiation on the nuclear location of the $a b l, b c r$ and $c$-myc genes in human leukemic cells, Leuk. Res. 24 (2000), 233-241.

[2] E. Bártová, S. Kozubek, M. Kozubek et al., Nuclear topography of the c-myc gene human leukemic cells, Gene 244 (2000), 111.

[3] F. Bahrmam, S. Wu, F. Ödberg, B. Lüsher and L.G. Larsson, Postranslation regulation of Myc function in response to phorbol ester/interferon- $\gamma$-induced differentiation of v-Myctransformed U-937 monoblasts, Blood 93 (1999), 3900-3912.
[4] K.E. Brown, J. Baxter, D. Graf, M. Markenschlager, A.G. Ficher, Dynamic repositioning of genes in the nucleus of lymphocytes preparing for cell division, Molecular Cell 3 (1999), 207-217.

[5] T. Cremer, S. Dietzel, R. Eils, P. Lichter and C. Cremer, Chromosome territories, nuclear matrix filaments and interchromatin channels: a topological view on nuclear architecture and function, in: Kew Chromosome Conference, 1995, pp. 6381.

[6] J.A. Croft, J.M. Bridger, S. Boyele, P. Perry, P. Teague and W.A. Bickmore, Differences in the localization and morphology of chromosomes in human nucleus, J. Cell Biol. 145 (1999), 1119-1131.

[7] A.K. Csink and S. Henikoff, Genetic modification in heterochromatin associations and nuclear organizations in Drosophila, Nature 381 (1996), 529-531.

[8] H. van Dekken, A. van Rotterdam, R.R. Jonker, H.T.M. van der Voort, G.J. Brakenhoff and J.G.J. Bauman, Spatial topography of a pericentromeric region (1Q12) in hemopoietic cells studied by in situ hybridization and confocal microscopy, Cytometry 11 (1990), 570-578.

[9] G. Diaz, A. Zucca, M.D. Setzu and C. Cappai, Chromatin pattern by variogram anylysis, Micros. Res. Technique 39 (1997), 305-311.

[10] F. Farabegoli, C. Ceccareli, D. Santini et al., Chromosome 1 aneusomy with 1 p36 under-representation is related to histological grade, DNA aneuploidy, high c-erb-2 and loss of bcl-2 expression in ductal breast carcinoma, Int. J. Cancer 69 (1996), 381-385.

[11] M. Ferguson and D.C. Ward, Cell cycle dependent chromosomal movement in pre-mitotic human T-lymphocyte nucleit, Chromosoma 101 (1992), 557-565.

[12] J. Ferreira, G. Paolella, C. Ramos and A.I. Lamond, Spatial organization of large-scale chromatin domains in the nucleus: magnified view of single chromosome territories, J.Cell Biol. 139 (1997), 1597-1610.

[13] Ch. Höfers, P. Baumann, G. Hummer, T.M. Jovin and D.J. Arndt-Jovin, The localization of chromosome domains in human interphase nuclei. Three-dimensional distance determinations of fluorescence in situ hybridization signals from confocal laser scanning microscopy, Bioimaging 1 (1993), 96-106.

[14] R. Hulspas, A.B. Houtsmuller, P.J. Krijtenburg, J.G.J. Bauman and N. Nanninga, The nuclear position of pericentromeric DNA of chromosome 11 appears to be random in $\mathrm{G}_{0}$ and non-random $\mathrm{G}_{1}$ human lymphocytes, Chromosoma 103 (1994), 286-292.

[15] A. de Klein, G. van den Kessel, G. Grosveld et al., A cellular oncogene is translocated to the Philadelphia chromosome in chronic myelocytic leukemia, Nature 300 (1982), 765-767.

[16] M. Kozubek, S. Kozubek, E. Lukášová et al., High-resolution cytometry: Automated 2-D and 3-D analysis of hybridization dots in interphase cell nuclei using multi-color FISH and CCD microscopy, Cytometry 36 (1999), 279-293.

[17] S. Kozubek, E. Lukášová, J. Amrichová et al., Distortion of nuclear topology in different preparation methods for FISH, Analytical Biochemistry, in press.

[18] S. Kozubek, E. Lukášová, A. Marečková et al., The topological organization of chromosomes 9 and 22 in cell nuclei has 
a determinative role in the induction of $\mathrm{t}(9 ; 22)$ translocations and in the pathogenesis of $\mathrm{t}(9 ; 22)$ leukemias, Chromosoma $\mathbf{1 0 8}$ (1999), 426-435.

[19] S. Kozubek, E. Lukášová, L Rýznar, M. Kozubek, A. Lišková and V. Kroha, Chromatin structure and its cell cycle kinetics in intact and irradiated cell nuclei, in: Fundamentals for the Assessment of Risks from Environmental Radiation, Ch. Baustark Khan, S. Kozubek and G. Horneck, eds, NATO Science Series, Kluwer Academic Publishers B.V., 1999, pp. 231-243.

[20] S. Kozubek, E. Lukášová, L. Rýznar et al., Distribution of ABL and BCR genes in cell nuclei of normal and irradiated lymphocytes, Blood 89 (1997), 4537-4545.

[21] E. Lukášová, S. Kozubek, M. Kozubek et al., Chromosomes participating in translocations typical of malignant haemoblastosis are also involved in exchange aberrations induced by fast neutrons, Rad. Res. 151 (1999), 375-384.

[22] E. Lukášová, S. Kozubek, M. Kozubek, J. Kjeronská, L. Rýznar, J. Horáková, E. Krahulcová and G Horneck, Localisation and distance between $\mathrm{ABL}$ and BCR genes in interphase nuclei of bone marrow cells of control donors and patients with chronic myeloid leukaemia, Hum. Genet. 100 (1997), 525-535.

[23] L. Manuelidis, Individual interphase chromosome domains revealed by in situ hybridization, Hum. Genet. 71 (1985), 291294.

[24] T. Mattefeld, Nonlinear deterministic analysis of tissue texture: a stereological study on mastopathic and mammary cancer tissue using chaos theory, Journal of Microscopy 85 (1997), 4766.

[25] L. Parreira, M. Telhada, C. Ramos, R. Hernandez, H. Neves and M. Carmo-Fonseca, The spatial distribution of human immunoglobulin genes within the nucleus: evidencefor gene topography independent of cell type and transcriptional activity, Hum. Genet. 100 (1997), 588-594.
[26] L. Pillus and M. Grustein, Chromatin structure and epigenetic regulation in yest, in: Chromatin Structure and Gene Expresion, S.C.R. Elgin, ed., IRL Press (Oxford University Press), 1995, pp. 123-146.

[27] M. Puech and F. Giroud, Standardisation of DNA quantitation by image analysis: quality control of instrumentation, Cytometry 36 (1999), 11-17.

[28] N. Sadoni, S. Langer, Ch. Fauth, G. Bernardi, T. Cremer, B.M. Turner and D. Zink, Nuclear organization of mammalian genomes: polar chromosome territories build up functionally distinct higher order compartments, J. Cell Biol. 146 (1999), 1211-1226.

[29] M. Skalníková, S. Kozubek, E. Lukášová et al., Spatial arrangement of genes, centromeres and chromosomes in cell nuclei of different types of human blood cells and its changes during the cell cycle, differentiation and after irradiation, Chromosome Research, in press.

[30] B.W. Steward, Mechanisms of apoptosis: Integration of genetic, biochemicals, and cellular indicators, J. Natl. Cancer Institute 86 (1994), 1286-1295.

[31] P.J. Verschure, I. van der Krann, E.M.M. Manders and R. van Driel, Spatial relationship between transcription sites and chromosomes territories, J. Cell Biol. 147 (1999), 13-24.

[32] W.R. Walter, G.B. Singh and S.A. Krawetz, MARs mission update, Biochem. Bioph. Res. Communications 242 (1998), 419422.

[33] G. van de Wouver, B. Weyn, P. Scheundres, W. Jacob, E. van Marck and D. van Dyck, Wavelets as chromatin texture descriptors for automated identification of neoplastic nuclei, J. Microsc. 197 (2000), 25-35. 


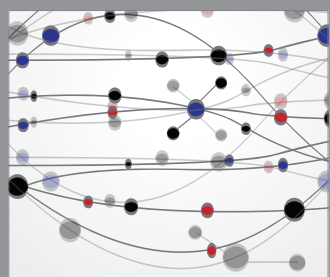

The Scientific World Journal
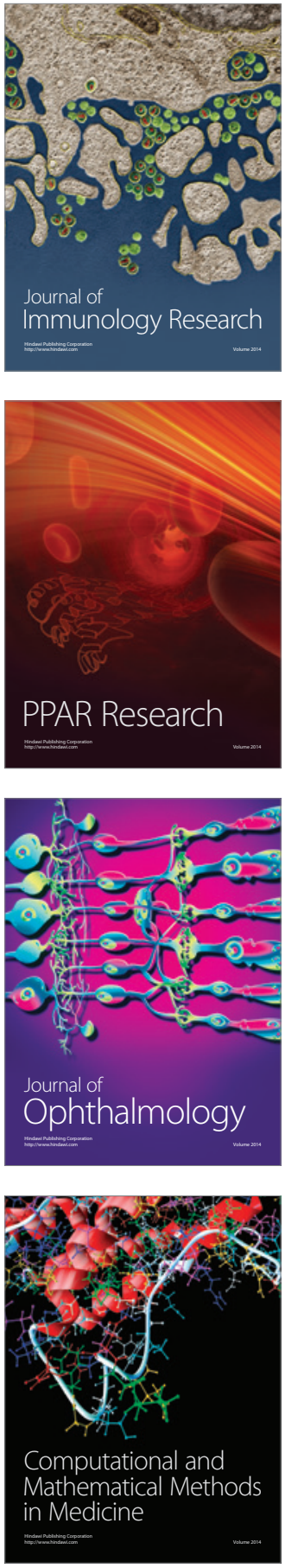

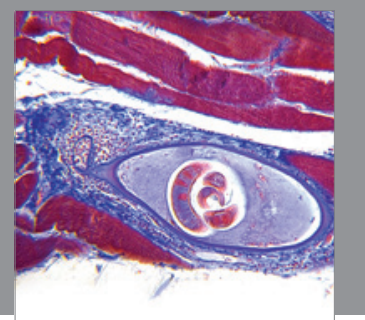

Gastroenterology

Research and Practice
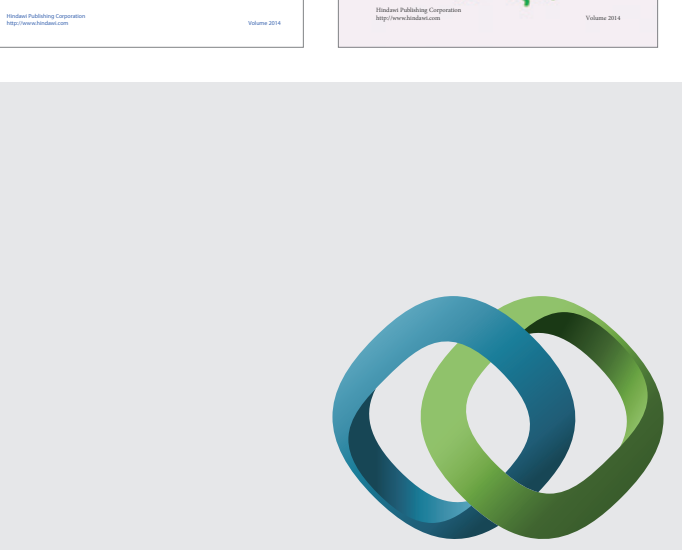

\section{Hindawi}

Submit your manuscripts at

http://www.hindawi.com
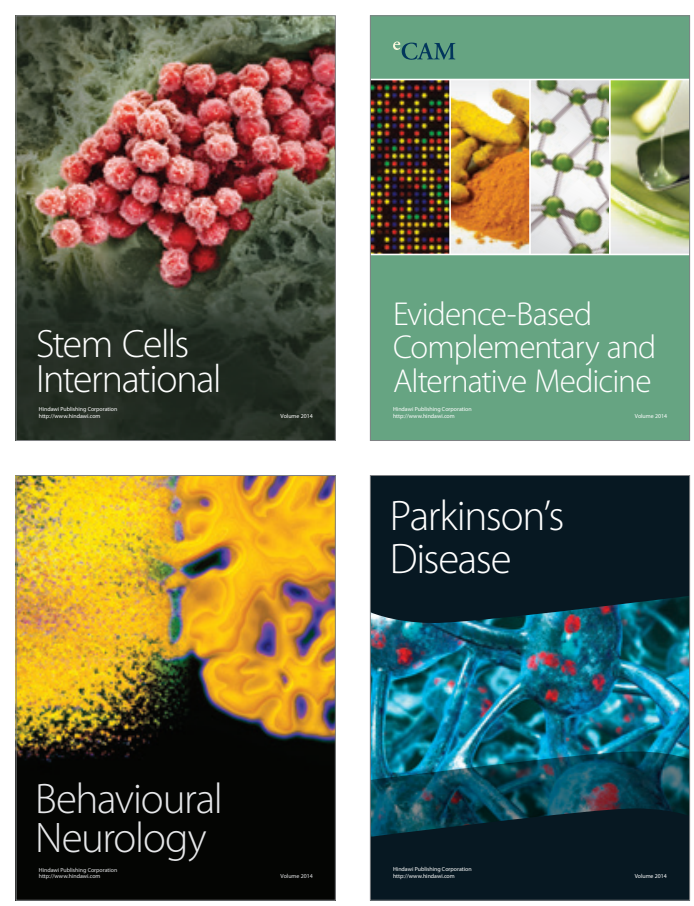

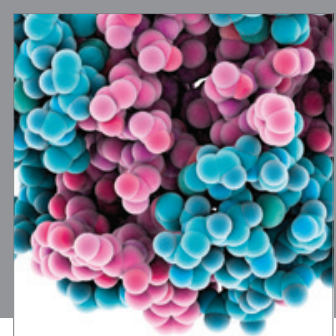

Journal of
Diabetes Research

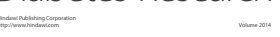

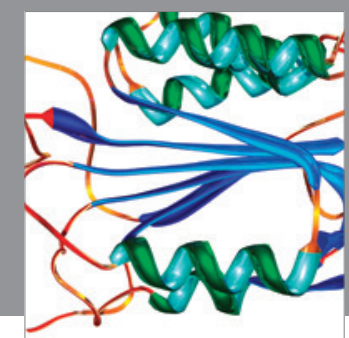

Disease Markers
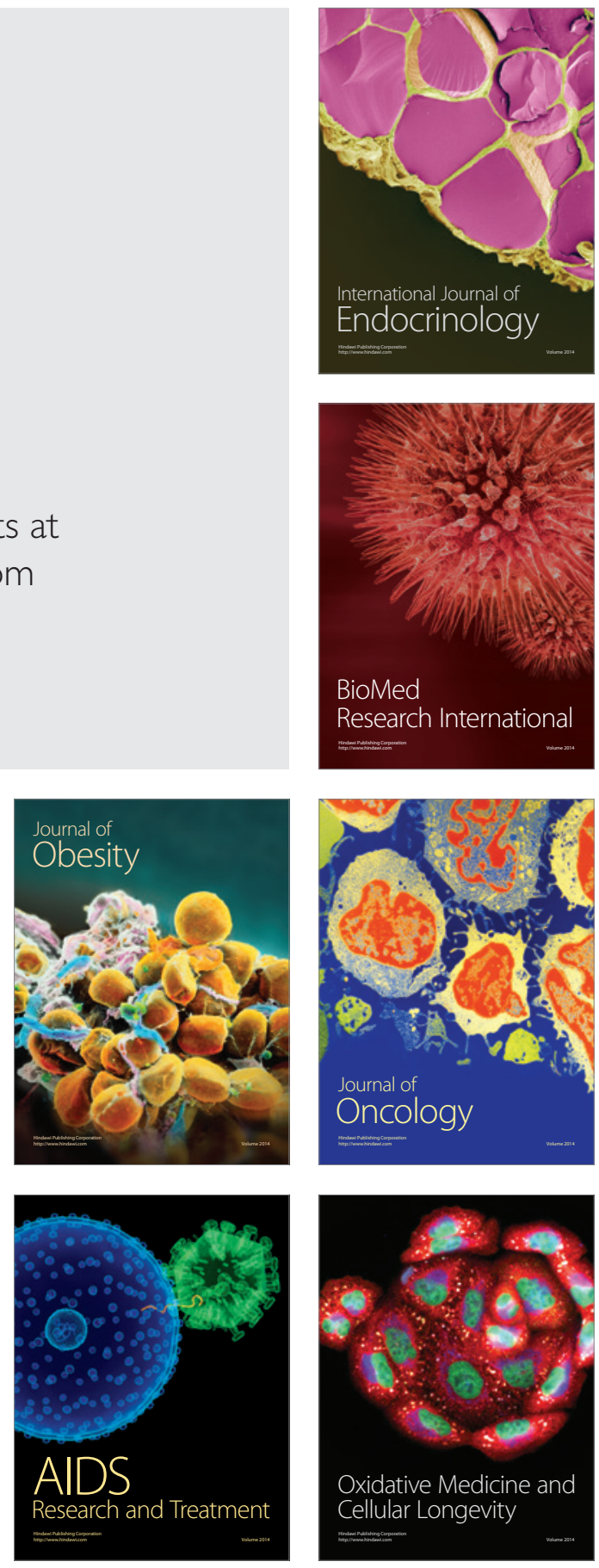